\title{
Towards a Workflow Management Approach for Health Monitoring of Bridges
}

\author{
Cesar Garita ${ }^{1}$ and Giannina Ortiz ${ }^{2}$ \\ ${ }^{1}$ Costa Rica Institute of Technology, School of Computer Science, \\ ${ }^{2}$ Costa Rica Institute of Technology, School of Construction Engineering, \\ Cartago 159-7050, Costa Rica \\ \{cesar,gortiz\}@itcr.ac.cr
}

\begin{abstract}
In brief, Structural Health Monitoring (SHM) refers to the storage and analysis of large amounts of sensor data associated to specific variables that indicate the condition of a civil structure, e.g. a bridge on a national highway. SHM analysis tasks typically involve the integration of different tools which may be highly heterogeneous, autonomous and physically distributed among different organizations. This paper introduces a scientific workflow management approach to model and execute strategic SHM queries that require proper integration of data generated by different software components.
\end{abstract}

Keywords: Structural health monitoring, bridges, workflow, data integration.

\section{Introduction}

Structural Health Monitoring (SHM) is a process consisting in the periodic recording of data generated by sensors associated to parameters that adequately reflect the performance and the condition of a civil structure [1]. This raw data is then processed to obtain valuable information for planning of maintenance activities, increase safety, verify hypothesis about the structure and, in general, to expand the knowledge about the behavior of the structure being monitored. Proper monitoring of civil structures such as bridges contributes to prevent the socio-economic consequences that may be caused by a damaged structured.

In the particular case of bridges, SHM systems should ideally support integrated decision-making processes through functionalities such as: user friendly web interfaces, GIS (Geographic Information System) services, distributed monitoring units, data management features, historic knowledge bases, expert systems, and integration of bridge inventory systems [2].

Although, there are some systems available in the market offering integrated solutions with many of these capabilities, in practice, individual institutions working on SHM have developed different systems and used a wide variety of tools depending on their specific objectives and approaches (see [3]). For an effective development of SHM policies, different institutions and professionals including civil engineers, university labs, transportation ministries, and local government units (municipalities), 
need to share and analyze large amounts of SHM-related information generated by heterogeneous and distributed systems and tools. This scenario lays out specific information integration requirements that can be met by Collaborative Networks (CNs) framework and in particular, by workflow management components.

This paper explores some SHM scenario requirements and introduces the application of scientific workflow management technologies for information integration in this field.

\subsection{Related Work}

There exist several studies regarding the state-of-the-art of SHM of bridges (see [4],[5]). These works summarize established and emerging technologies, practices and projects in the field. Other reports focus mostly on commercially available systems and their functionalities for SHM support, including specific systems such as BRIMOS and SHM Live [6], [7]. These systems offer a variety of services and functionalities for on-line monitoring that come "off-the-shelf" with a proprietary infrastructure. Other specific approaches for data management systems in SHM can be found in [8], [9], [10]. Most of these initiatives are based on a centralized relational database approach for gathering, storing and analyzing data.

On the other hand, the Collaborative Network $(\mathrm{CN})$ paradigm has been successfully applied to similar complex domains (e.g. industrial manufacturing, tourism value-added service provision, virtual laboratories) in which different entities that are physically distributed, autonomous and heterogeneous, need to collaborate in order to achieve common goals based on computer networks [11]. In fact, an early conceptual paper on Virtual Enterprises (VEs) mentions how this manifestation of CNs can be applied in a "building a bridge scenario" during the preparation of the call-for-tenders for a construction project [12].

Nevertheless, the application of specific CN models and tools in SHM, such as distributed business processes or workflow management techniques in order to define, execute and coordinate tasks that must be achieved by network entities, remains an open issue. In [3], a ontology-based mediator system is proposed for semantic integration of different tools and systems in SHM; this mediator is seen as a "router" that suggests specific tools to the user following a general workflow (planning, monitoring, data preparation, evaluation). However, this approach uses this workflow as a reference and does not consider generic workflow management features.

In this paper, we present a novel approach for data integration based on generic definition, execution and monitoring of scientific workflows that support different data analysis scenarios in SHM. The proposed approach is being developed in the context of the e-Bridge 2.0 project as described in the following section.

\section{The e-Bridge 2.0 General Approach}

The main goal of the e-Bridge 2.0 Project - Integrated System for Performance Assessment of Bridge Structures, is to develop a prototype of a system for solving 
strategic queries regarding the performance of bridges. This project is carried out at the Costa Rica Institute of Technology (TEC) as part of an e-Science Program bringing together engineers from different schools including Construction Engineering, Electronics, Computer Science, Industrial Production Engineering and Forest Engineering. The main components to be developed or integrated within e-Bridge 2.0 include:

- Reliability model: a probabilistic model to predict structural behavior of bridges using variables that have an impact on potential structural failures.

- Technical information system: integration of data from on-going national inventory of bridges and their technical characteristics.

- Sensors systems: development of sensors networks for SHM of bridges to measure variables such as vibration, deflection and deformation.

- GIS: web-based geospatial information system to properly assess environmental aspects associated to national bridges.

- Information integration system: a single entry-point for users in order to access and analyze strategic information generated by other components.

The implementation approach followed in the project promotes the use of existing open-source technologies and tools from multidisciplinary domains based on a peerto-peer communication architecture. This approach foresees integration of other e-science tools regarding for instance: simulation environments, 3D visualization tools and high-performance computing clusters. Please notice that in this "open environment", each component may use different data models and software tools. However, in order to solve strategic queries regarding high-level SHM scenarios, information from these heterogeneous sources needs to be seamlessly integrated from a single interface for end-users. The following section provides some examples of these requirements and scenarios.

\section{Requirements Analysis for Information Integration}

In order to identify general functional requirements for the e-Bridge platform, two main kinds of workshops were organized:

1. Internal workshops. A first workshop was organized with the university colleagues working on the project in order to achieve a common internal perspective on the main requirements for the project. This workshop allowed the identification of main personas (user roles) and system functionalities from an internal point of view.

2. External workshops. Several other workshops were organized with external potential users of the platform including collaborators from the Ministry of Transportation, Ministry of Agriculture, Ministry of Health, National Road Council, municipality councils, and civil engineers. These external workshops delivered new potential functionalities and allowed us to validate the results of the internal workshop. 
Some of the strategic queries that were identified through these activities include:

- Real-time failure detection based on different types of sensors.

- Validation of structural models (real vs. theoretical bridge models).

- Generation of bridge reliability rankings.

- Analysis of remaining lifetime for given bridges.

- Identification of failure tendencies by structure types.

- Lists of bridges by maintenance priorities.

- Alternative traffic routes in case of bridge failures.

- Vulnerability (risk) analysis of bridges according to environmental factors.

This analysis allowed the identification of requirements for the general platform and more specifically for the Information Integration System. For this system, we concluded that there are two main types of strategic queries that need to be covered:

1. Predefined queries: solving specific strategic queries for high-level end users e.g. a minister who asks: which are the most important bridges that need urgent maintenance in the country?

2. Analysis queries: solving specific technical queries for experts and engineers that need to perform SHM tasks or queries of a more scientific / experimental nature, e.g. can this bridge support an 80 -ton truck load at $60 \mathrm{~km} / \mathrm{h}$ speed?

The first type involves common important queries that are relatively fixed or welldefined but that need to be solved through configurable integration of different components. For instance, in the case of "real-time failure detection", the following interaction sequence among platform actors can be outlined:

1. Sensors System detects a strain measurement value out of the normal range for a given bridge beam and sends an alarm in real time to the Information Integration System.

2. Information Integration System sends an alert to the Geographic Information System so that the event is properly reflected in the GIS layers.

3. Information Integration System records the event in the bridge Technical Information System.

4. Information Integration System sends an alarm SMS/email message to operators responsible for bridge maintenance.

5. Bridge maintenance operators access the Information Integration System interface with a dashboard for global bridge monitoring, providing access to detailed information about the event.

6. Bridge maintenance operators enter the GIS from the Information Integration System and analyze possible actions depending on the bridge location.

Currently, the components involved in this scenario are managed by TEC, but in the near future, they may be managed by different organizations, for example: the bridge inventory may be managed by the Ministry of Transportation, the Geographic Information System may be hosted in the cloud by a GIS service provider, the bridge monitoring dashboard may be accessed by National Road Council, and the 
Information Integration System and the Reliability Model may be hosted at TEC or other university.

The second type above involves queries that relate to a flexible "problem-solving environment" (e.g. similar to a virtual laboratory) in which construction engineers need to experiment, test hypothesis and answer questions in different ways. An example of this kind of queries will be given in the next section. In both cases, queries require the integration of data from different tools that may be located in different organizations.

Based on the analysis both kinds of strategic queries, a scientific workflow approach is suggested to properly model and support these requirements. This workflow approach is described next.

\section{Scientific Workflow Management Scenarios}

According to the Workflow Management Coalition, a workflow is "the automation of a business process, in whole or part, during which documents, information or tasks are passed from one participant to another for action, according to a set of procedural rules"[13]. Workflows represent a prevailing approach for service-oriented integration since they provide the "glue for distributed services, which are owned and maintained by multiple organizations" [14]. When workflows are applied to support large-scale scientific applications, they are called scientific workflows. These scientific workflows set forward specific challenges in relation to traditional businessoriented workflow management, such as the need to handle large amount of data, intense computational tasks, and more data provenance and "interactive steering" features for scientists and engineers [15]. Furthermore, workflow management technologies have been extensively used to support Collaborative Networks scenarios involving distributed, heterogeneous and autonomous entities [11],[16],[17]. Certainly, other approaches for coordination of these entities are possible, including agent-based technologies, coordination languages, and GRID services. Nevertheless, the workflow-based architecture was chosen for e-Bridge since it represents a simple, common, flexible and user-friendly approach for service integration. In particular, scientific workflows tools allow the modeling and execution of SHM analysis tasks in a way that can be easily adopted by engineers and scientists from different disciplines with some technical training.

In this context, based on the requirements analysis presented in the previous section, a workflow management approach has been adopted for information integration in e-Bridge 2.0. To this end, several business process / workflow tools have been compared and evaluated including Bonita, Taverna, Bizagi, and jBPM among others. As a result of this evaluation, Taverna is being currently used to model and execute preliminary workflows in this project.

Taverna is a Java-based open-source workflow management system that offers a set of tools to define and execute scientific workflows [18]. Taverna provides a desktop application that allows scientists and engineers to easily define and execute workflows without the need of advanced software development skills. Taverna 


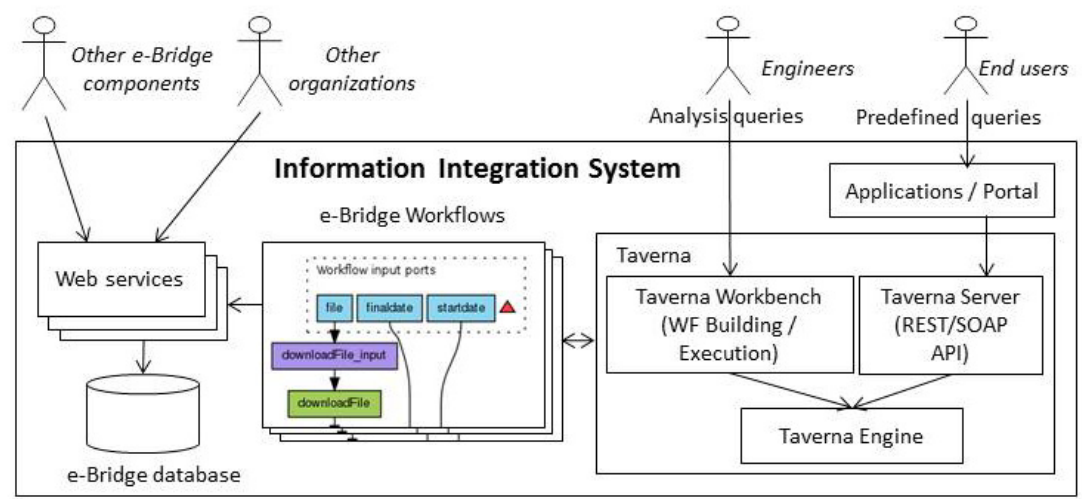

Fig. 1. General architecture of the Information Integration System

workflows can also be edited and executed on the web and invoked from the command line in batch mode. Workflows are represented as directed acyclic graphs using a dataflow language called SCUFL (Simple Conceptual Unified Flow Language). Nodes in these graphs are called processors which can be regarded as mathematical functions mapping a set of input data to a set of output data. Processors can be connected through data links to achieve function composition. Finally, Taverna allows the integration of many different software components through SOAP or REST web services.

The proposed general architecture for the Information Integration System of eBridge 2.0 is depicted on Fig. 1. This figure describes how the system architecture supports analysis queries for engineers as well as predefined queries for end-users, using Taverna workbench and server tools respectively. In this way, e-Bridge workflows can be created and executed orchestrating web services related to other eBridge components and potential organizations.

The current prototype of system includes basic Taverna workflows which allows construction engineers to seamlessly orchestrate distributed web services to carry on sensor data acquisition, database storage and data visualization (see Fig. 2). In brief, the sample workflow presented in this figure basically collects sensor data as a file from a remote data collection unit (e.g. FTP server) associated to a bridge, processes the file, stores the data in the local database, and finally formats and exports data sets that can be visualized on a web browser (see Fig. 3).

Workflows can be properly edited by engineers by using other existing web services and connecting them accordingly in a graphical environment. Workflows can also be executed from Java applications that will offer strategic queries for SHM. Currently available web services have been developed using SOAP with Java on Ubuntu Linux operating system and PostgreSQL as database engine. 


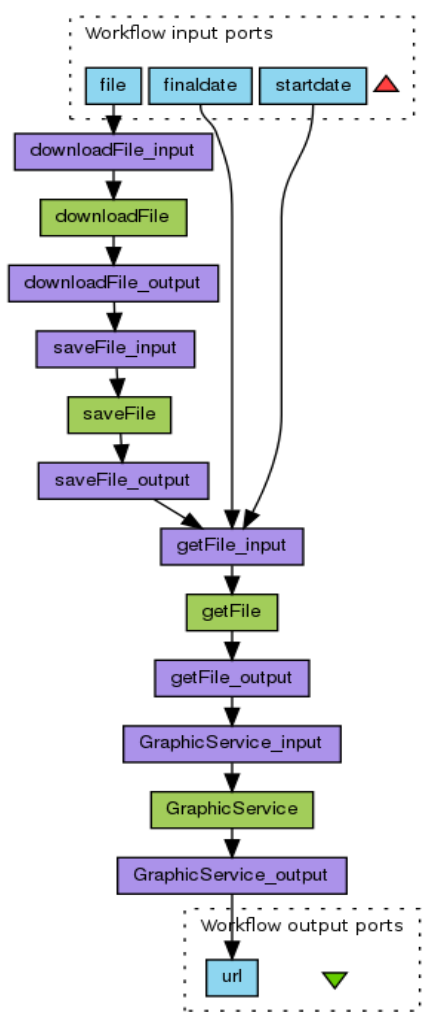

Fig. 2. Sample partial Taverna workflow for sensors data analysis

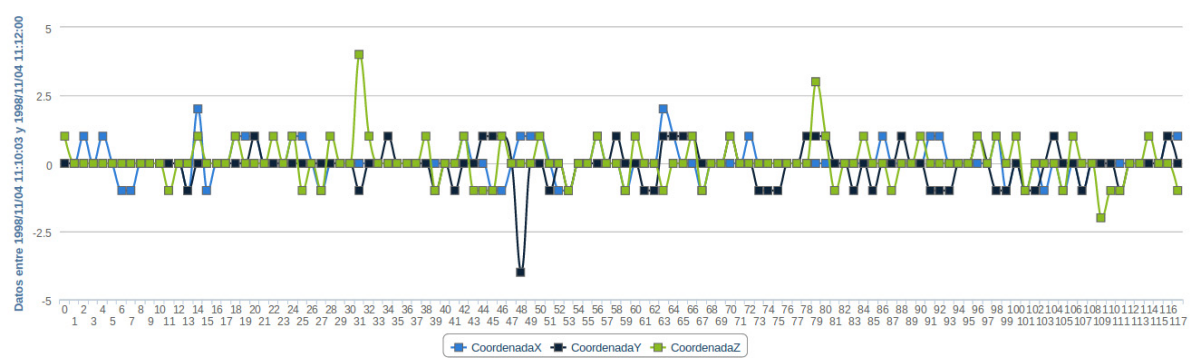

Fig. 3. Visualization of sensor data for bridge vibration variables

\section{Conclusions and Future Work}

This paper described how a scientific workflow management approach can be applied to support information integration scenarios in the specific domain of structural health monitoring of bridges. In summary, through the workflow-based information integration component, e-Bridge 2.0 end-users will be able to solve general strategic queries as well as tailored experimentation/analysis tasks regarding structural 
monitoring of bridge behavior. The proposed service-oriented workflow approach allows the seamless integration of sensors, GIS, reliability model and technical data, which may be physically distributed over different organizations.

Future work within the project includes the specification of more integration scenarios involving other components and organizations, development and integration of web-services according to these scenarios, and development of web applications.

Acknowledgements. The authors of this paper wish to acknowledge the valuable contributions from all colleagues and partners of the e-Bridge 2.0 project.

\section{References}

1. Glisic, B., Inaudi, D.: Fibre Optic Methods for Structural Health Monitoring. John Wiley \& Sons (2007)

2. Wenzel, H.: Health Monitoring of Bridges. Wiley (2009)

3. Küng, J., Sonnleitner, E., Stumptner, R., Kosorus, A.H., Anderlik, S.: Utilizing Ontologies to Integrate Heterogeneous Decision Support Systems. In: Wenzel, H. (ed.) Industrial Safety and Life Cycle Engineering - Technologies/Standards/Applications (2013)

4. Ahlborn, T.M., Shuchman, R., Sutter, L.L., Brooks, C.N., Harris, D.K., Burns, J.W., Endsley, K.A., Evans, D.C., Vaghefi, K., Oats, R.C.: The State-of-the-Practice of Modern Structural Health Monitoring for Bridges: A Comprehensive Review. Michigan Technological University (2010)

5. Glisic, B., Inaudi, D., Casanova, N.: SHM process - lessons learned in 250 SHM Projects. In: 4th International Conference on Structural Health Monitoring on Intelligent Infrastructure (SHMII-4), Zurich, Switzerland (2009)

6. Dong, Y., Song, R.: Bridges Structural Health Monitoring and Deterioration Detection Synthesis of Knowledge and Technology. University of Alaska Fairbanks (2010)

7. Brimos Project, http://www. brimos.com

8. Koo, K.Y., Battista, N.D.: SHM Data Management System Using MySQL Database with MATLAB and Web Interfaces. In: 5th International Conference on Structural Health Monitoring of Intelligent Infrastructure (SHMII-5), Cancún, México (2011)

9. Tas, N.C., Dejori, C.R.M., Neubauer, C.: Bridge Sensor Mart: A Flexible and Scalable Data Storage and Analysis Framework for Structural Health Monitoring. In: 5th International Conference on Bridge Maintenance, Safety and Management, Philadelphia, USA (2010)

10. Inaudi, D., Glisic, B., Vurpillot, S.: Database structures for the management of monitoring data. In: Structural Health Monitoring Workshop, Winnipeg, Canada (2002)

11. Camarinha-Matos, L.M., Afasarmanesh, H.: Collaborative networks: a new scientific discipline. Journal of Intelligent Manufacturing 16 (2005)

12. Camarinha-Matos, L.M., Afsarmanesh, H.: The Virtual Enterprise Concept. In: Camarinha-Matos, L.M., Afsarmanesh, H. (eds.) Infrastructures for Virtual Enterprises Networking Industrial Enterprises, pp. 3-14. Kluwer Academic (1999)

13. Workflow Management Coalition, http: //www.wfmc.org/

14. Barker, A., van Hemert, J.: Scientific Workflow: A Survey and Research Directions. In: Wyrzykowski, R., Dongarra, J., Karczewski, K., Wasniewski, J. (eds.) PPAM 2007. LNCS, vol. 4967, pp. 746-753. Springer, Heidelberg (2008) 
15. Chen, J., van der Aalst, W.M.P.: On scientific workflows. Technical Committee on Scalable Computing Newsletter 9 (2007)

16. Afsarmanesh, H., Garita, C., Camarinha-Matos, L.M., Lima, C.: Workflow Support for Management of Information in PRODNET II. In: 5th International Workshop on Intelligent Manufacturing Systems - IMS 1998, pp. 49-54 (1998)

17. Garita, C., Unal, O., Afsarmanesh, H., Hertzberger, L.O.: Building a Virtual Laboratory for Scientific Experimentation in Molecular Biology. In: Camarinha-Matos, L.M., Afsarmanesh, H. (eds.) Processes and Foundations for Virtual Organizations, pp. 181-190. Kluwer Academic (2003)

18. Taverna Project, http: //www . taverna. org.uk/ 